\title{
Observational analysis on role of acupuncture in duration of functional recovery in Bell's Palsy: A pilot study
}

\author{
Jaya Satyal ${ }^{1,2}$, Ayush Chandra ${ }^{3}$, Avinash Chandra ${ }^{1 *}$ and Pradeep $\mathrm{KC}^{1,2}$ \\ ${ }^{1}$ Annapurna Neurological Institute and Allied Sciences, Kathmandu, Nepal \\ ${ }^{2}$ Nardevi Ayurvedic Hospital, Kathmandu, Nepal \\ ${ }^{3}$ Tianjin Medical University, PR China
}

\begin{abstract}
Bell's palsy is a peripheral unilateral facial weakness (lower motor neuron) paralysis with an acute onset yet unknown cause. Bell's palsy accounts for almost three quarters of peripheral facial palsies and the annual incidence is about 15 to 30 patients per 100000 with equal numbers of males and females. Acupuncture has been a safe clinical practice. It has a low risk of adverse events. Besides having a good safety margin, it is also a very cost-effective therapy. To our knowledge, acupuncture is yet not popular in therapeutics in Nepal and this study is first of its kind to describe the therapeutic value of acupuncture. With the usage of acupuncture, we found that the duration of recovery from severe Bell's palsy was quite rapid than expected.
\end{abstract}

Abbreviations: TCM: Traditional Chinese Medicine; HB grade/ scale: House-brackmann grade/scale.

\section{Introduction}

Bell's palsy is a peripheral unilateral facial weakness (lower motor neuron) paralysis with an acute onset yet unknown cause. Bell's palsy accounts for almost three quarters of peripheral facial palsies and the annual incidence is about 15 to 30 patients per 100000 with equal numbers of males and females [1]. There is no predilection for either side of the face. Recurrence rate of Bell's palsy is about $8 \%$ [2]. Because of the unclear etiology of Bell's palsy, there has been a longstanding controversy on the type of treatment protocol to be used. Alternatives include corticosteroids, antiviral drugs, acupuncture, and physiotherapy. Corticosteroids are commonly used as treatment of Bell's palsy with the not yet proven concept of inflammation as a pathogenesis. It is also claimed that about $71 \%$ of untreated patients with Bell's palsy will completely recover and $84 \%$ will have complete or near normal recovery. About $16-20 \%$ people will have persistent moderate to severe weakness, facial contracture, or synkinesis [3,4]. Study shows that there is a significant short-term and long-term positive treatment effect of corticosteroids in patients with Bell's palsy [5]. The various adverse effects of corticosteroids also comes with its concomitant usage. Acupuncture has been a safe clinical practice. It has a low risk of adverse events [6]. Besides having a good safety margin, it is also a very cost effective therapy, and thus one of the commonly used treatments of Bell's palsy and now gaining popularity globally [6]. Despite good safety margin, cost effectiveness and good outcome, convincing evidence for the efficacy of acupuncture in Bell's palsy treatment is lacking because of lack of good study designs, adequate sample sizes and treatment reports. The management of Bell's palsy would include steroid, antivirals, surgical decompressions $[7,8]$. The duration of recovery of Bell's palsy on average goes on to several weeks to several months (even till year). A research done on more than a hundred of patients showed that with house-brackmann (HB) scale of III, it took in average of 6 months to recover fully functionally [9]. Thus, in view of this, we came up with a protocol to combine both pharmacotherapy with acupuncture in Bell's palsy patients, with the aim of comparing the duration in the recovery of the affected facial nerve. We categorized the severity of facial palsy according to the HB stages and combined acupuncture along with prednisolone and compared the functional recovery and compared with the stages in $\mathrm{HB}$ scale. To our knowledge, acupuncture is yet not popular in therapeutics in Nepal and this study is first of its kind to describe the therapeutic value of acupuncture.

\section{Materials and methods}

\section{Subjects}

This was a prospective analysis. Patients who visited our hospital were first seen by the neurologist and then by the acupuncture specialist and when there was agreement for the diagnosis of bell's palsy, then the patient was prescribed medicine according to standard dosing criteria and acupuncture. Data of patients visiting on out-patient basis were collected and follow-up was done accordingly. Proper informed consent was obtained in the written form from the patient. The patient data collected was kept strictly confidential and for study purpose use only. The study was conducted according to the Helsinki declaration. Patients and enrolment consisted of inclusion and exclusion criteria.

\section{Inclusion criteria and exclusion criteria}

Only patients with Bell's palsy were included in our study. Paralysis of unilateral facial nerve only were included. Age inclusion was kept between 8 and 80 years old. Facial nerve paralysis onset period within 15 days. While, exclusion criteria was pregnant ( $1^{\text {st }}$ Trimester) or

*Correspondence to: Avinash Chandra, Dept. of Neurology, Annapurna Neurology and Allied Sciences, Maitighar, Kathmandu, E-mail: chandraavi@gmail.com

Key words: bell's palsy, acupuncture, house-brackmann, observational analysis

Received: March 19, 2020; Accepted: April 08, 2020; Published: April 15, 2020 
other neurological disease that may interfere with course and nature of this disease. Other chronic diseases like diabetes, poorly controlled hypertension, renal disease, hepatic disease, duodenal ulcer, glaucoma, $\mathrm{TB}$, or any other condition that is a risk of being influenced by the study. Those that were not willing to consent and/or participate in the study were also excluded from the study. However, our study did not discriminate between newly diagnosed Bell's palsy or recurrent bell's palsy.

\section{Interventions}

Drug therapy: Prednisolone as $1 \mathrm{mg} / \mathrm{kg}$ body weight with the tapering dose for patients presenting in the out-patient within 7 days with a total treatment time around 20 days.

Acupuncture therapy: Based on previous studies, the acupuncture points used were Dicang (ST4), Jiache (ST6), Yangbai (GB14), Xiaguan (ST7), Taiyang (EXHN5), Quanliao (SI18) and Yifeng (TE17) on the affected side, and Hegu (LI4) bilaterally. Qualified acupuncturist did the acupuncture. Shallow puncturing was used at facial acupoints and routine puncturing was used at other acupoints within $72 \mathrm{~h}$ after onset of Bell's palsy. Yifeng (TE17), Hegu (LI4) was punctured 0.5-1.0 cun, the others will be punctured 0.1-0.3 cun. Deep puncturing was done with penetrative needling, which will be used from Dicang (ST4) to Jiache (ST6) and from Taiyang (EX-HN5) to Quanliao (SI18) 2-3 cun, and other routine puncturing [10]. Filiform needles $(33-49.5 \mathrm{~mm}$, $0.32 \mathrm{~mm}$ ) was used with moderate stimulation to get an acupuncture sensation, and the needles were retained for 30 minutes, once a day, five times a week, for a total period of four weeks. The locations of those points are defined in 1993 by the World Health Organization (WHO), Regional Office for the Western Pacific [10]. Physiotherapy facial exercises associated either with mirror or electromyogram biofeedback, and other nerve stimulation techniques were used as well. Acupuncture was performed by the specialists in Chinese traditional medicine (TCM) with a 6 years course in TCM and certified by the TCM board in China, and with a license to practice acupuncture as awarded by the council in Nepal.

\section{Outcomes measures}

Functional measure of facial palsy was conducted, and the outcome measures were assessed with the House-Brackmann scoring systems. The outcome was measured at time points 7 days, 14 days, 21 days, and 28 days.

\section{Statistics}

A sample of nearly 100 participants was collected. Among the participants, house-brackmann scale was noted in all the participants on the day of diagnosis (named as pretreatment), day7, as the $7^{\text {th }}$ day of starting intervention (medicine and acupuncture), day 14 as the $14^{\text {th }}$ day of intervention, and similarly day 21 and day 28 . All the data were analyzed descriptively using software SPSS version 20. Among the outcome variables, ANOVA was applied. The frequency of HB grade was measured in pre and post treatment. Similarly, median was compared. A statistical software SPSS 20 was used. Among the outcome measure, HB scale was recorded and grouped as on day 7 , day 14 , day 21 and day 28. Intervention acupuncture and outcome was measured using one-way ANOVA.

\section{Result}

A total of 99 of the participants were included in the study. Frequency table was constructed which showed that the higher frequency of $\mathrm{HB}$ grade of 6 (most severe deficit) was present in the beginning in patients with Bell's palsy. HB grade 6 was quite frequent (49\%) and only few patients had lower HB grade of 3 (mild deficit). It was as low as (6.1\%). After the $7^{\text {th }}$ day of acupuncture, the severity decreased rapidly and the change in HB grade was observed. Facila palsy improved significantly and $\mathrm{HB}$ grade of 6 (most severe deficit) was only in few (1\%) of participants which after $28^{\text {th }}$ day of intervention, decreased to 0 (normal grade). The severity of HB grade decreased further after the $28^{\text {th }}$ day of acupuncture. The mild deficit, that is HB grade of 3 was seen in few participants only (6.1\%), while the majority recovered very well and had no deficit. HB grade of 0 (normal functioning of nerve) had become more frequent (66\%) after the acupuncture on day 28. (As shown in Table 1). Interestingly, the right side of facial palsy was observed to be more frequent (64\%) in our study. The intervention and outcome were analyzed with ANOVA, which showed a strong statistical significance at $\mathrm{p} \leq 0.0(\mathrm{p}=0.00)$ in all the intervention groups (acupuncture done at day 7, 14, 21, 28). The sample was tested for homogeneity and it showed normal distribution of data. Illustrated in.

\section{Discussion}

The effects of acupuncture can be improved by the different manipulations performed and by the experienced acupuncturist. In this study, we found that acupuncture had a beneficial effect on Bell's palsy patient. It relieves the physical impairment and thus improves the functionality. The focal effect of acupuncture on paralysis could be attributed, in part, to the local effects of acupuncture in stimulating nerve fibers in the skin and muscle [11]. The psychosomatic effects of acupuncture on the autonomic nerve system could be contributed by regulating Qi and thereby improving the functional outcome [12]. The rapid recovery of deficit in our patient was attributed mainly due to the rapid intervention (within few days of onset) of acupuncture in our patient despite majority of patient had severe facial nerve palsy. It's generally seen that among Bell's palsy cases, $70 \%$ of the cases improve before 6 months (but longer than 4 weeks) [4,13]. while about $30 \%$ remain residual palsy and other sequelae like contractures and synkinesis [14]. Some researchers opined that the recovery from the disease depended mostly on the severity of the peripheral nerve lesion but not the disease itself [15]. Until now, almost all the research studies comprising acupuncture usage in Bell's palsy come from the mainland China or few places of South Korea [16]. The strength of our study is that we were strict in our inclusion criteria and we did not discriminate

Table 1. Showing the comparison of frequency of HB grade at the time of presentation (preintervention) and 28th day of acupuncture in Bell's palsy participants

\begin{tabular}{|l|c|c|c|}
\hline \multicolumn{2}{|c|}{ Preintervention } & \multicolumn{3}{|c|}{} \\
\hline \multirow{4}{*}{ Valid } & 3.00 & Frequency & Percent \\
\cline { 2 - 4 } & 4.00 & 6 & 6.1 \\
\cline { 2 - 4 } & 5.00 & 13 & 13.1 \\
\cline { 2 - 4 } & 6.00 & 31 & 31.3 \\
\hline \multirow{3}{*}{ Day 28 } & Total & 49 & 49.5 \\
\hline \multirow{4}{*}{ Valid } & & 99 & 100.0 \\
\cline { 2 - 4 } & .00 & & \\
\cline { 2 - 4 } & 1.00 & 66 & 66.7 \\
\cline { 2 - 4 } & 2.00 & 13 & 13.1 \\
\cline { 2 - 4 } & 3.00 & 6 & 10.1 \\
\cline { 2 - 4 } & 4.00 & 3 & 3.1 \\
\cline { 2 - 4 } & 5.00 & 1 & 1.0 \\
\hline
\end{tabular}


between the first or recurrent Bell's palsy and it was a prospective study. The weakness of our study was that we had relatively smaller number of participants $(n=100)$, however considering it a pilot study, this number of participants can be considered satisfactory. The other weakness was that we did not have comparative study with the control or sam acupuncture as well as we could not filter out the confounding factor(s).

\section{Conclusion}

Acupuncture is pragmatic therapeutic option and apparently with no known side effects. Acupuncture can be a useful tool that can be applied for in the treatment of Bell's palsy for the rapid recovery. The residual deficit not only can be reduced, but also the recovery time can be significantly reduced by acupuncture in Bell's palsy. The mechanism is mainly due to a balance of muscle activation in response to the electrical stimulation of the acupuncture needles.

A further elaborative prospective study with larger number of participants and randomized trial will be needed to show the better efficacy of acupuncture in Bell's palsy.

\section{References}

1. Morales DR, Donnan PT, Daly F, Staa TV, Sullivan FM (2013) Impact of clinical trial findings on Bell's palsy management in general practice in the UK 2001-2012: interrupted time series regression analysis. BMJ Open 3: e003121 [Crossref]

2. Gilden DH (2004) Clinical practice. Bell's Palsy. N Engl J Med 351: 1323-1331. [Crossref]

3. Holland NJ, Weiner GM (2004) Recent developments in Bell's palsy. BMJ 329: 553557. [Crossref]

4. Peitersen E (2002) Bell's palsy: the spontaneous course of 2,500 peripheral facial nerve palsies of different etiologies. Acta Otolaryngol Suppl 10: 4-30. [Crossref]
5. Engstrom M, Berg T, Stjernquist-Desatnik A (2008) Prednisolone and valaciclovir in Bell's palsy: a randomised, double-blind, placebo-controlled, multicentre trial. Lancet Neurol 7: 993-1000. [Crossref]

6. Zhao L, Zhang FW, Li Y (2011) Adverse events associated with acupuncture: three multicentre randomized controlled trials of 1968 cases in China. Trials 12: 87 [Crossref]

7. Grogan PM, Gronseth GS (2001) Practice parameter: Steroids, acyclovir, and surgery for Bell's palsy (an evidence-based review): report of the quality standards subcommittee of the american academy of neurology. Neurology 56: 830-836. [Crossref]

8. Ravikumar A, Singh P, Batish VK (1999) Facial Palsy - Treatment Options. Med J Armed Forces India 55: 41-44. [Crossref]

9. Ferreira M, Firmino-Machado J, Marques EA, Santos PC, Simoes AD, et al. (2016) Prognostic factors for recovery in Portuguese patients with Bell's palsy. Neurol Res 38: 851-856. [Crossref]

10. Lim S (2010) WHO Standard Acupuncture Point Locations. Evid Based Complement Alternat Med 7: 167-168. [Crosref]

11. White A, Cummings TM, Filshie J (2018) An introduction to Western medical acupuncture.

12. Kondo T, Kawamoto M (2014) Acupuncture and moxibustion for stress-related disorders. Biopsychosoc Med 8: 1-7. [Crossref]

13. Adour KK (1982) Current concepts in neurology: diagnosis and management of facial paralysis. N Engl J Med 307: 348-351. [Crossref]

14. Yamamoto E, Nishimura H, Hirono Y (1988) Occurrence of sequelae in Bell's palsy Acta Otolaryngol Suppl 446: 93-96. [Crossref]

15. Volk GF, Klingner C, Finkensieper M, Witte OW, Guntinas-Lichius (2013) Prognostication of recovery time after acute peripheral facial palsy: a prospective cohort study. BMJ Open 3: e003007 [Crossref]

16. Li P, Qiu T, Qin C (2015) Efficacy of acupuncture for bell's palsy: A systematic review and meta-analysis of randomized controlled trials. PLoS One 10: 0121880 [Crossref]

Copyright: (C2020 Satyal J. This is an open-access article distributed under the terms of the Creative Commons Attribution License, which permits unrestricted use, distribution, and reproduction in any medium, provided the original author and source are credited. 